\title{
Nonlinear Response Characteristics of Electrostrictive Material Sensors
}

\author{
Qian-Peng Li, ${ }^{1}$ Jia Xu, ${ }^{1,2}$ Guo-Song Feng, ${ }^{3}$ and Zhi-Wen $\mathrm{Zhu}^{2 *}$ \\ ${ }^{1}$ Department of Mechanics, Tianjin University, \\ 92 Weijin Road, Nankai District, Tianjin 300072, China \\ ${ }^{2}$ Tianjin Key Laboratory of Nonlinear Dynamics and Control, Tianjin University, \\ 92 Weijin Road, Nankai District, Tianjin 300072, China \\ ${ }^{3}$ Beijing Institute of Spacecraft Environment Engineering, Beijing 100094, China
}

(Received April 16, 2018; accepted April 15, 2019)

Keywords: electrostrictive materials, sensors, stochastic nonlinear dynamics, bifurcation

In this study, the nonlinear response characteristics of electrostrictive material sensors subjected to stochastic excitation were investigated. Van der Pol nonlinear differential items were developed to interpret the hysteretic phenomena of electrostrictive materials. The hysteretic constitutive models of electrostrictive materials were proposed, and the nonlinear dynamic model of electrostrictive material sensors subjected to stochastic excitation was developed. The expression of the dynamic response of the system was obtained, and the bifurcation conditions and chaos characteristics of the system were determined. Finally, the effects of system parameters on the dynamic characteristics of the system were analyzed. The simulation results show that there were bifurcation phenomena in the system, which can be suppressed by adjusting the parameters. The stochastic noise intensity had an important effect on the system dynamical response, and the stochastic resonance phenomenon occured with the variation in stochastic noise intensity. The results of this study were helpful for the optimal design and improvement of electrostrictive material sensors.

\section{Introduction}

Electrostrictive materials are kinds of smart materials that can convert electrical and mechanical energies into each other. They have the advantages of high conversion efficiency, high energy density, large electrodeformation, small viscoelastic lag, and high response speed. ${ }^{(1-3)}$ They have great potential applications in microactuators, artificial muscles, and robots. A highly sensitive sensor is an important component in modern electronic products. An electrostrictive material sensor is a new kind of sensor. It has high accuracy and self-generating characteristics, and is used widely where the power supply is very difficult to be obtained.

Many researchers have studied the characteristics of electrostrictive materials. Kuang performed a thorough study of electroelastic and magnetoelastic solids by using the variational principle. $^{(4)}$ The electrostrictive constitutive equation, electric field volume force, and

*Corresponding author: e-mail: zhuzhiwen@tju.edu.cn https://doi.org/10.18494/SAM.2019.2274 
mechanical boundary conditions were discussed in detail. ${ }^{(4)}$ The axisymmetric stresses in hollow electrostrictive cylinders were discussed by Jiang and Gao. ${ }^{(5)}$ In 2006, Beom et al. studied the gradual formation of the insulation crack tip in electrostrictive materials. ${ }^{(6)}$ Sunder and Newnham determined the electrostrictive constants of low-dielectric-constant materials. ${ }^{(7)}$ Cheng et al. studied the electrostrictive properties of copolymers. ${ }^{(8)}$ Della Schiava et al. discussed the enhanced figures of merit for a high-performing actuator in electrostrictive materials. ${ }^{(9)}$ The effects of interface diffusion on the strain and stress stability of particulate reinforced electrostrictive materials were determined by Li and Wang. ${ }^{(10)}$

Although many achievements in electrostrictive materials were reported in previous years, theoretical results on the dynamic characteristics of electrostrictive material sensors are few. The nonlinear response characteristics of electrostrictive material sensors subjected to stochastic excitation are examined in this study. Van der Pol nonlinear differential items are developed to interpret the hysteretic phenomena of electrostrictive materials. The hysteretic constitutive models of electrostrictive materials are proposed, and the nonlinear dynamic model of electrostrictive material sensors subjected to stochastic excitation is developed.

\section{Hysteretic Nonlinear Model of Electrostrictive Material Sensors}

The strain-stress curve of electrostrictive materials is shown in Fig. 1. Obviously, hysteretic phenomena are observed in the curve. A new differential item is introduced to describe the hysteretic phenomena as

$$
\sigma=a_{1} \varepsilon+a_{2} \varepsilon^{3}+a_{3} \varepsilon^{5}+\left(a_{4} \varepsilon+a_{5} \varepsilon^{2}+a_{6} \varepsilon^{3}+a_{7} \varepsilon^{4}\right) \dot{\varepsilon}
$$

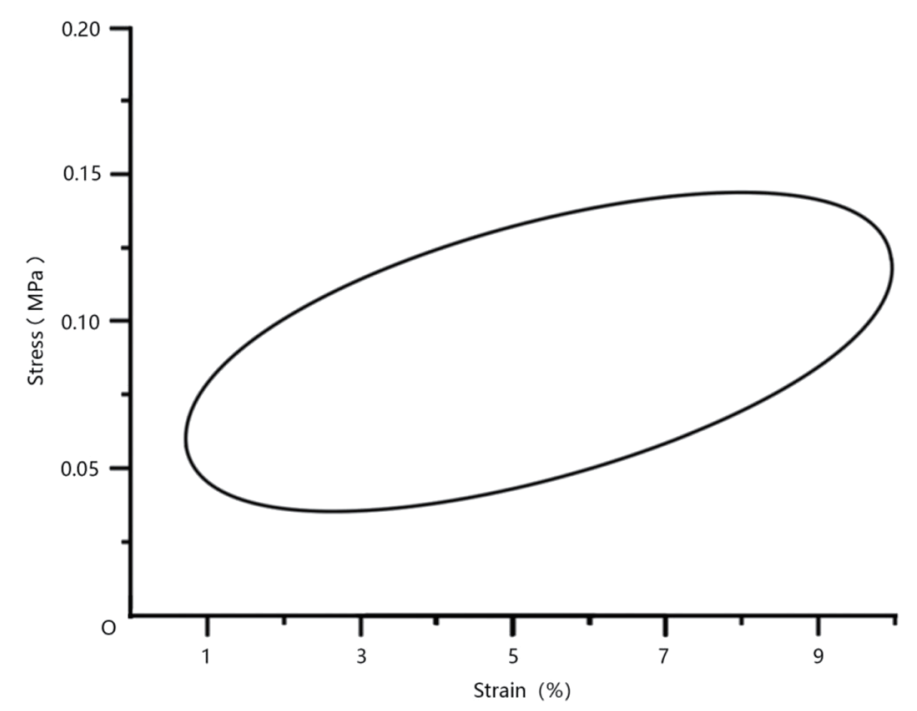

Fig. 1. Strain-stress curve. 
In Eq. (1), only the hysteretic characteristic of the strain-stress curve of electrostrictive materials is considered. In this study, a new nonlinear differential item, which is developed from a Van der Pol differential item, is introduced to describe the hysteretic nonlinear characteristics of the strain-stress curve of electrostrictive materials in a differentiable function so as to obtain more theoretical results. The partial least-squares regression software SIMCA-P is used to determine the relationships among strain and stress. The partial least-squares regression method reduces the sum of squared errors to find the best match of data and is usually used for curve fitting.

The model developed to describe the strain-stress curve of electrostrictive materials indicates that the final relationship between stress and strain is

$$
\sigma=k_{1} \varepsilon+k_{2} \varepsilon^{3}+k_{3} \varepsilon^{5}+\left(k_{4} \varepsilon+k_{5} \varepsilon^{2}+k_{6} \varepsilon^{3}+k_{7} \varepsilon^{4}\right) \dot{\varepsilon}
$$

The result of the forecast test to Eq. (1) is shown in Fig. 2, where the red line represents the real data and the black line represents the forecast value. Equation (1) can describe the real curve well.

\section{Dynamic Characteristics of the System}

Considering the complex characteristics of the materials, we introduce Hamilton's principle to the dynamic modeling of the system. The Hamilton function can be presented as

$$
S=T-U+W,
$$

where $T$ is the kinetic energy, $T=\frac{1}{2} \int_{0}^{L} \rho A\left(\frac{\partial u}{\partial t}\right)^{2} d x ; U$ is the potential energy; $W$ is the power of external force, $\delta W=\int_{0}^{L} \delta u N d x$. Moreover, $\rho$ is the density, $V$ is the volume, $E$ is the elastic modulus, and $A$ is the cross-sectional area.

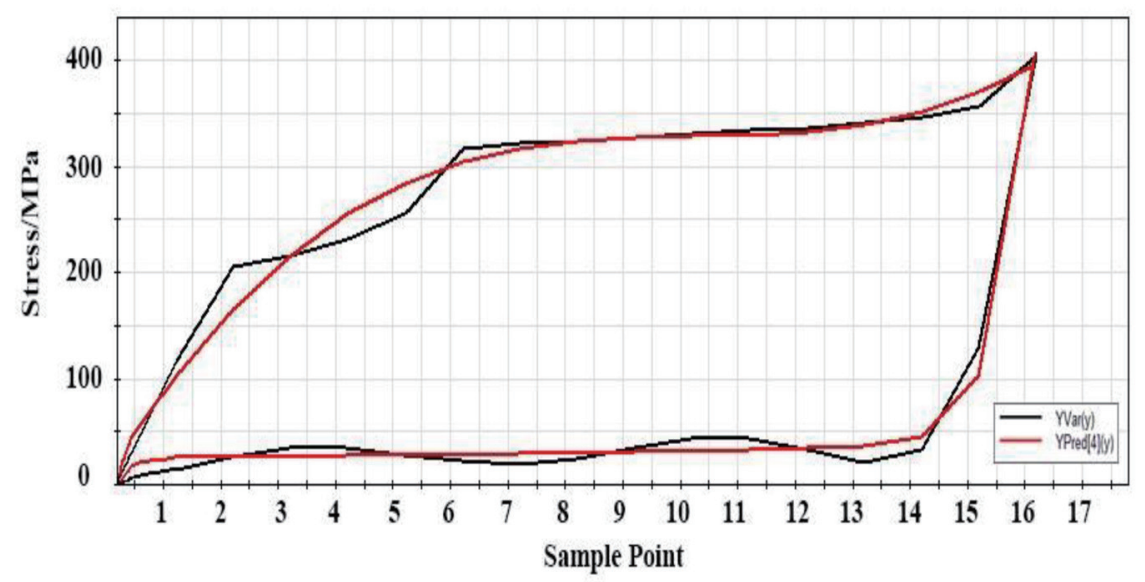

Fig. 2. (Color online) Results of the forecast test. 
According to Hamilton's principle,

$$
\delta S=\int_{t_{1}}^{t_{2}} \delta(T-U+W) d t=0 .
$$

Thus, the nonlinear dynamic model of the system can be shown as

$$
m \frac{\partial^{2} u}{\partial t^{2}}+\left[c+\int_{0}^{L}\left(a_{6} u+a_{7} u^{2}\right) d x\right] \frac{\partial u}{\partial t}-a_{1} \frac{\partial^{2} u}{\partial x^{2}}-a_{2} \frac{\partial^{4} u}{\partial x^{4}}-a_{3} \frac{\partial^{2} u}{\partial x^{2}} \int_{0}^{L}\left(\frac{\partial u}{\partial x}\right)^{2} d x=\frac{1}{6} E A \frac{\partial^{2} u}{\partial x^{2}}
$$

Then, the dynamic equation of system response can be solved from Eq. (5) by the Galerkin's method as

$$
\ddot{q}+c_{1} q+c_{2} q^{3}+c_{3} q^{5}+\left(2 \eta+c_{4} q^{2}\right) \dot{q}=e q \varsigma(t),
$$

where $2 \eta=\frac{c}{m}, c_{1}=\frac{a_{1} \pi^{4}}{m}, c_{2}=\frac{3 a_{3} \pi^{8}}{4 m}, c_{3}=\frac{3 a_{5} \pi^{8}}{4 m}, c_{4}=\frac{5 a_{7} \pi^{12}}{8 m}, e$ is the intensity of stochastic excitation, and $\varsigma(t)$ is the Guass white noise, whose intensity is $2 D$.

We can obtain the Ito equation corresponding to Eq. (6) using the stochastic average method as

$$
d H=m(H) d t+\bar{\sigma}(H) d B(t),
$$

where $B(t)$ is the standard Wiener process and $m(H)$ and $\bar{\sigma}(H)$ are the drift and diffusion coefficients, respectively.

$$
\begin{gathered}
m(H)=\left(\frac{D e^{2}}{c_{1}}-\eta\right) H-\frac{c_{2}}{2 c_{1}} H^{2}+\frac{c_{3}}{2 c_{1}^{2}} H^{3}-\frac{7 c_{4}}{8 c_{1}^{4}} H^{5} \\
\bar{\sigma}^{2}(H)=\frac{D e^{2} H^{2}}{c_{1}}
\end{gathered}
$$

The averaged Fokker-Planck-Kolmogorov (FPK) equation of Eq. (7) is

$$
\frac{\partial f}{\partial t}=-\frac{\partial}{\partial H}[m(H) f]+\frac{1}{2} \frac{\partial^{2}}{\partial H^{2}}\left[\sigma^{2}(H) f\right],
$$

where $f$ is the stationary probability density of system response.

$$
f(H)=\bar{A} H^{-\frac{2 \eta c_{4}}{D e^{2}}} \exp \left[\frac{c_{1}}{D e^{2}} H-\frac{c_{2}}{4 D e^{2} c_{4}^{2}} H^{2}+\frac{5 c_{3}}{12 D e^{2} c_{4}^{3}} H^{3}\right]
$$


Here, $\bar{A}$ is a normalization constant.

The numerical results of the system response are presented in Figs. 3-5, where $\eta=0.2, c_{1}=0.5$, $c_{2}=0.2, c_{3}=0.09, c_{4}=0.05$, and $D=0.4$.

According to Figs. $3-5$, we obtain the following results:

1) $p=0$ and $q=0$ when $H=0$, given that $H=\frac{1}{2} p^{2}+\frac{1}{2} c_{1} q^{2}$. Thus, the trivial solution $H=0$ corresponds to the origin point $(0,0)$ in the SPD map.

2) In the variation process of the system parameters, the system response can jump from the balance point to periodic orbits under external excitation, which causes the bifurcation of the system.

The simulation experimental results of the system response are shown in Fig. 6, where $\eta=0.2$, $c_{1}=0.5, c_{2}=0.2, c_{3}=0.09, c_{4}=0.05$, and $D=0.4$.

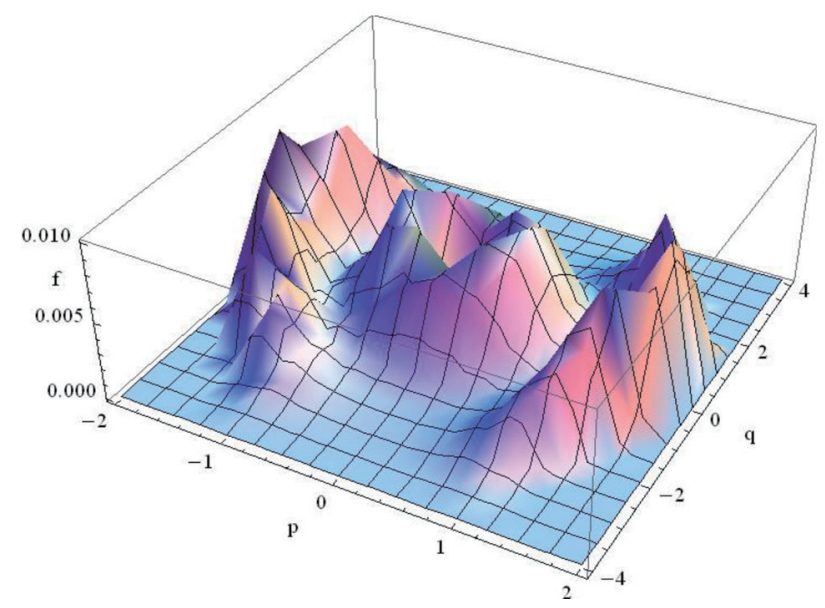

Fig. 3. (Color online) Stationary probability density when $e=0.2$.

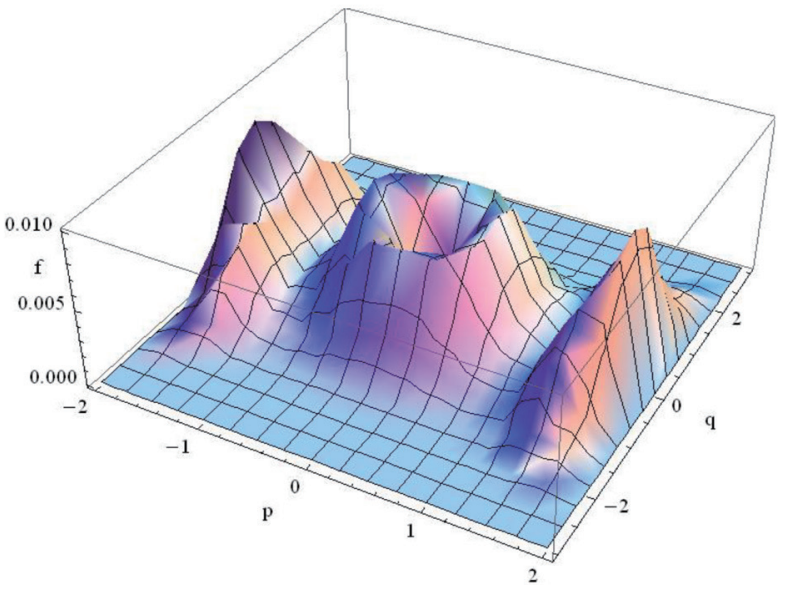

Fig. 4. (Color online) Stationary probability density when $e=0.5$.

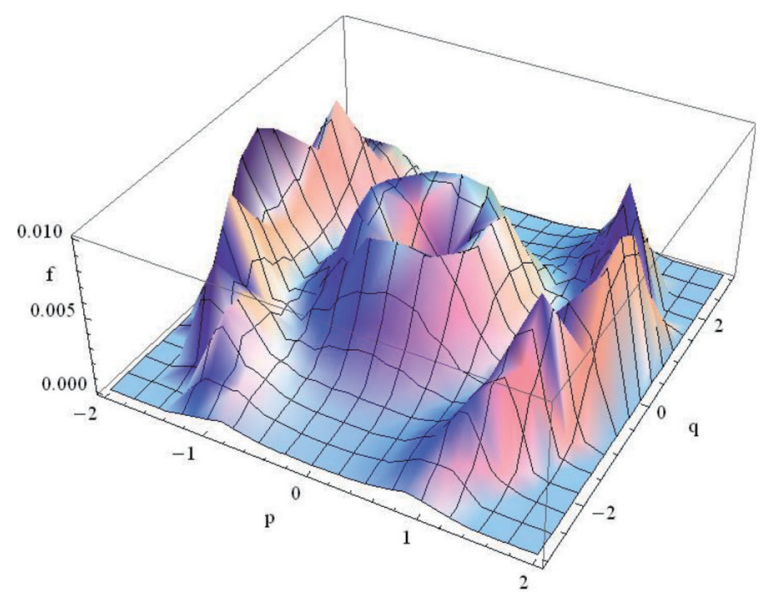

Fig. 5. (Color online) Stationary probability density when $e=0.9$. 


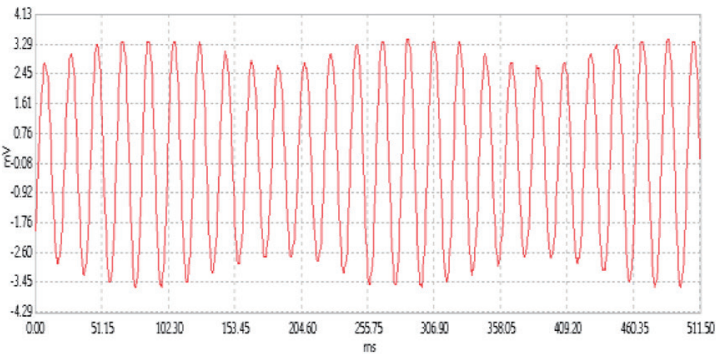

(a)

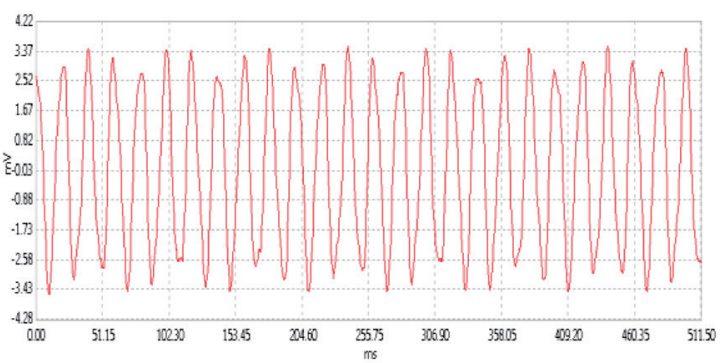

(c)

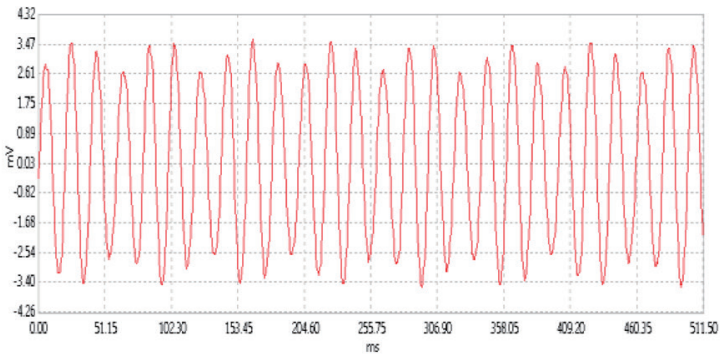

(b)

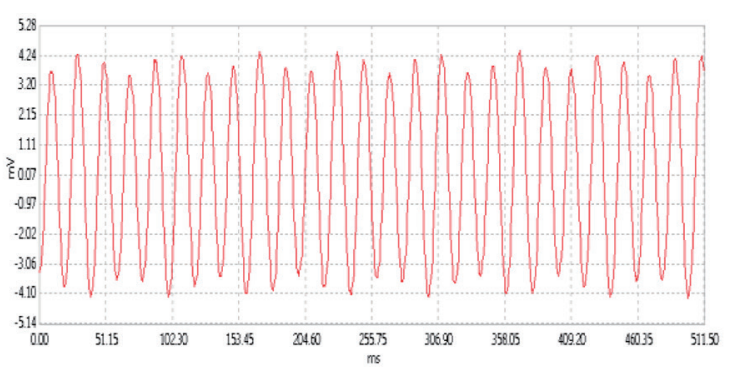

(d)

Fig. 6. (Color online) Experimental results of the system response. (a) $e=0.1$, (b) $e=0.3$, (c) $e=0.5$, and (d) $e=0.9$.

The vibration amplitude of the system is shown as the output voltage. As shown by the figures, the stochastic noise intensity has an important effect on the system dynamical response. When the intensity of stochastic excitation is low, it is slightly effective for vibrating; with the increase in the intensity of stochastic excitation, the system output increases. However, with the further increase in the intensity of stochastic excitation, the system vibration decreases again. The system vibration increases significantly when the intensity of stochastic excitation increases. This phenomenon is called stochastic resonance.

\section{Conclusions}

The nonlinear dynamic response of electrostrictive material sensors in stochastic excitation is described in this paper. Nonlinear differential items are introduced to interpret the hysteretic phenomena of electrostrictive materials, and the nonlinear dynamic model of electrostrictive material sensors is developed. The dynamic response of the system is obtained. The results of numerical experiments show that the stochastic noise intensity has an important effect on the system dynamical response. When the stochastic noise intensity reaches a certain value, stochastic resonance occurs. The system safety will be strengthened by a control strategy. The results of this paper are helpful for the application of electrostrictive material sensors in engineering fields. 


\section{Acknowledgments}

The authors gratefully acknowledge the support of the Natural Science Foundation of China (NSFC) through Grant Nos. 11872266 and 51875396, Chinese Aviation Science Foundation through Grant No. 2016ZA48001, the Tianjin Research Program of Application Foundation and Advanced Technology through Grant No. 16JCYBJC18800, and Cast-Bisee 511 through Grant No. CAST-BISEE2017-006.

\section{References}

1 J. Biggs, K. Danielmeier, and J. Hitzbleck: Angew. Chem. Int. Ed. 52 (2013) 9409.

2 P. Brochu and Q. Pei: Macromol. Rapid. Commun. 31 (2010) 10.

3 L. J. Romasanta, M. A. Lopez-Manchado, and R. Verdejo: Prog. Polym. Sci. 41 (2015) 188.

4 J. B. Kuang: Eur. J. Mech. A Solids 3 (2008) 504.

5 Q. Jiang and C. F. Gao: Acta. Mech. 211 (2010) 309.

6 H. G. Beom, Y. H. Kim, and C. Cho: Int. J. Solids. Struct. 43 (2006) 6869.

7 V. Sundar and R. E. Newnham: Mater. Res. Bull. 31 (1996) 545.

8 Z. Y. Cheng, V. Bharti, and T. B. Xu: Sens. Actuators, A 90 (2001) 138.

9 N. Della Schiava, K. Thetpraphi, and M. Q. Le: Polymer 10 (2018) 263.

10 H. B. Li and X. Wang: Compos. Part B. Eng. 75 (2015) 319.

\section{About the Authors}

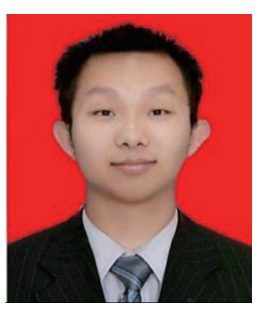

Qian-Peng Li received his B.S. in Engineering Mechanics from Kunming Polytechnic University, China, in 2017. Now, he is an M.S. student in Tianjin University. His current research interests focus on the nonlinear dynamics and bifurcation of smart structures.

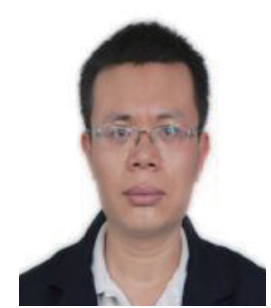

Jia Xu received his B.S., M.S., and Ph.D. degrees from Tianjin University, China, in 2003, 2005, and 2008, respectively. Since 2008, he has been an assistant professor at Tianjin University. His research interests are in the stochastic dynamics analysis and control of nonlinear systems.

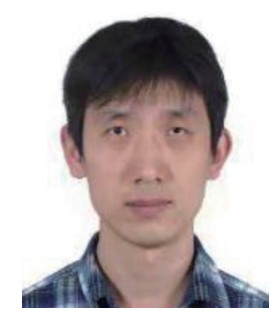

Guo-Song Feng received his B.S. degree from Beijing University of Aeronautics and Astronautics, China. Since 2009, he has been working at the Beijing Institute of Spacecraft Environment Engineering. His research interests are in the environmental simulation and testing of spacecraft dynamics. 
Zhi-Wen Zhu received his B.S. degree from Shanghai Jiaotong University, China, in 1996 and his M.S. and Ph.D. degrees from Tianjin University, China, in 2000 and 2003, respectively. From 2003 to 2005, he was a postdoctoral fellow at Tianjin University, China. In 2005, he worked as an assistant professor at Tianjin University, and then he became an associate professor at Tianjin University in 2010. His research interests are in the nonlinear dynamics and control of smart structures. 